\title{
Formalization of the Advanced Encryption Standard. Part $\mathbf{I}^{1}$
}

\author{
Kenichi Arai ${ }^{2}$ \\ Tokyo University of Science \\ Chiba, Japan
}

\author{
Hiroyuki Okazaki \\ Shinshu University \\ Nagano, Japan
}

Summary. In this article, we formalize the Advanced Encryption Standard (AES). AES, which is the most widely used symmetric cryptosystem in the world, is a block cipher that was selected by the National Institute of Standards and Technology (NIST) as an official Federal Information Processing Standard for the United States in 2001 [12. AES is the successor to DES [13], which was formerly the most widely used symmetric cryptosystem in the world. We formalize the AES algorithm according to 12. We then verify the correctness of the formalized algorithm that the ciphertext encoded by the AES algorithm can be decoded uniquely by the same key. Please note the following points about this formalization: the AES round process is composed of the SubBytes, ShiftRows, MixColumns, and AddRoundKey transformations (see [12]). In this formalization, the SubBytes and MixColumns transformations are given as permutations, because it is necessary to treat the finite field $\operatorname{GF}\left(2^{8}\right)$ for those transformations. The formalization of AES that considers the finite field $\operatorname{GF}\left(2^{8}\right)$ is formalized by the future article.

MSC: 68P25 94A60 03B35

Keywords: Mizar formalization; Advanced Encryption Standard (AES) algorithm; cryptology

MML identifier: AESCIP_1, version: 8.1.02 5.19.1189

The notation and terminology used in this paper have been introduced in the following articles: [5], [1], [13], [4, 6], [16], [14, [11, 7], 8], 15], [18], 2], 3], [9], [19], [17], and [10].

\footnotetext{
${ }^{1}$ This work was supported by JSPS KAKENHI 21240001 and 22300285.

${ }^{2}$ This research was presented during the 2012 International Conference on Foundations of Computer Science FCS'12 in Las Vegas, USA.

(C) 2013 University of Białystok CC-BY-SA License ver. 3.0 or later ISSN 1426-2630(Print), 1898-9934(Online)
} 


\section{Preliminaries}

Let us consider natural numbers $k, m$. Now we state the propositions:

(1) If $m \neq 0$ and $(k+1) \bmod m \neq 0$, then $(k+1) \bmod m=(k \bmod m)+1$.

(2) If $m \neq 0$ and $(k+1) \bmod m \neq 0$, then $(k+1) \operatorname{div} m=k \operatorname{div} m$.

(3) If $m \neq 0$ and $(k+1) \bmod m=0$, then $m-1=k \bmod m$.

(4) If $m \neq 0$ and $(k+1) \bmod m=0$, then $(k+1) \operatorname{div} m=(k \operatorname{div} m)+1$.

(5) $(k-m) \bmod m=k \bmod m$.

(6) If $m \neq 0$, then $(k-m) \operatorname{div} m=(k \operatorname{div} m)-1$.

Let $m, n$ be natural numbers, $X, D$ be non empty sets, $F$ be a function from $X$ into $\left(D^{n}\right)^{m}$, and $x$ be an element of $X$. Let us observe that the functor $F(x)$ yields an element of $\left(D^{n}\right)^{m}$. Let $m$ be a natural number, $X, Y, D$ be non empty sets, and $F$ be a function from $X \times Y$ into $D^{m}$. Let $y$ be an element of $Y$. Note that the functor $F(x, y)$ yields an element of $D^{m}$. Now we state the propositions:

(7) Let us consider natural numbers $m, n$, a non empty set $D$, and elements $F_{1}, F_{2}$ of $\left(D^{n}\right)^{m}$. Suppose natural numbers $i, j$. If $i \in \operatorname{Seg} m$ and $j \in \operatorname{Seg} n$, then $F_{1}(i)(j)=F_{2}(i)(j)$. Then $F_{1}=F_{2}$.

(8) Let us consider a non empty set $D$ and elements $x_{1}, x_{2}, x_{3}, x_{4}$ of $D$. Then $\left\langle x_{1}, x_{2}, x_{3}, x_{4}\right\rangle$ is an element of $D^{4}$.

(9) Let us consider a non empty set $D$ and elements $x_{1}, x_{2}, x_{3}, x_{4}, x_{5}$ of $D$. Then $\left\langle x_{1}, x_{2}, x_{3}, x_{4}, x_{5}\right\rangle$ is an element of $D^{5}$.

(10) Let us consider a non empty set $D$ and elements $x_{1}, x_{2}, x_{3}, x_{4}, x_{5}, x_{6}$, $x_{7}, x_{8}$ of $D$. Then $\left\langle x_{1}, x_{2}, x_{3}, x_{4}\right\rangle \frown\left\langle x_{5}, x_{6}, x_{7}, x_{8}\right\rangle$ is an element of $D^{8}$. The theorem is a consequence of (8).

(11) Let us consider a non empty set $D$ and elements $x_{1}, x_{2}, x_{3}, x_{4}, x_{5}, x_{6}$, $x_{7}, x_{8}, x_{9}, x_{10}$ of $D$. Then $\left\langle x_{1}, x_{2}, x_{3}, x_{4}, x_{5}\right\rangle \frown\left\langle x_{6}, x_{7}, x_{8}, x_{9}, x_{10}\right\rangle$ is an element of $D^{10}$. The theorem is a consequence of $(9)$.

(12) Let us consider a non empty set $D$ and elements $x_{1}, x_{2}, x_{3}, x_{4}, x_{5}, x_{6}$, $x_{7}, x_{8}$ of $D^{4}$. Then $\left\langle x_{1} \frown x_{5}, x_{2} \frown x_{6}, x_{3} \frown x_{7}, x_{4} \frown x_{8}\right\rangle$ is an element of $\left(D^{8}\right)^{4}$. The theorem is a consequence of (8).

(13) Let us consider a non empty set $D$, an element $x$ of $\left(D^{4}\right)^{4}$, and an element $k$ of $\mathbb{N}$. Suppose $k \in \operatorname{Seg} 4$. Then there exist elements $x_{1}, x_{2}, x_{3}, x_{4}$ of $D$ such that

(i) $x_{1}=x(k)(1)$, and

(ii) $x_{2}=x(k)(2)$, and

(iii) $x_{3}=x(k)(3)$, and

(iv) $x_{4}=x(k)(4)$. 
(14) Let us consider non empty sets $X, Y$, a function $f$ from $X$ into $Y$, and a function $g$ from $Y$ into $X$. Suppose

(i) for every element $x$ of $X, g(f(x))=x$, and

(ii) for every element $y$ of $Y, f(g(y))=y$.

Then

(iii) $f$ is one-to-one, and

(iv) $f$ is onto, and

(v) $g$ is one-to-one, and

(vi) $g$ is onto, and

(vii) $g=f^{-1}$, and

(viii) $f=g^{-1}$.

\section{State Array}

The array of AES-State yielding a function from Boolean ${ }^{128}$ into $\left(\left(\text { Boolean }^{8}\right)^{4}\right)^{4}$ is defined by

(Def. 1) Let us consider an element $i_{1}$ of Boolean ${ }^{128}$ and natural numbers $i, j$. Suppose $i, j \in \operatorname{Seg} 4$. Then $i t\left(i_{1}\right)(i)(j)=\operatorname{mid}\left(i_{1},\left(1+\left(i-{ }^{\prime} 1\right) \cdot 8\right)+\left(j-^{\prime}\right.\right.$ $\left.1) \cdot 32,\left(\left(1+\left(i-^{\prime} 1\right) \cdot 8\right)+\left(j-^{\prime} 1\right) \cdot 32\right)+7\right)$.

Now we state the propositions:

(15) Let us consider a natural number $k$. Suppose $1 \leqslant k \leqslant 128$. Then there exist natural numbers $i, j$ such that

(i) $i, j \in \operatorname{Seg} 4$, and

(ii) $\left(1+\left(i-^{\prime} 1\right) \cdot 8\right)+\left(j-^{\prime} 1\right) \cdot 32 \leqslant k \leqslant\left(\left(1+\left(i-^{\prime} 1\right) \cdot 8\right)+\left(j-^{\prime} 1\right) \cdot 32\right)+7$.

(16) Let us consider natural numbers $i, j, i_{0}, j_{0}$. Suppose

(i) $i, j, i_{0}, j_{0} \in \operatorname{Seg} 4$, and

(ii) it is not true that $i=i_{0}$ and $j=j_{0}$.

Then $\left\{k\right.$, where $k$ is a natural number : $\left(1+\left(i-^{\prime} 1\right) \cdot 8\right)+\left(j-^{\prime} 1\right) \cdot 32 \leqslant$ $\left.k \leqslant\left(8+\left(i-^{\prime} 1\right) \cdot 8\right)+\left(j-^{\prime} 1\right) \cdot 32\right\} \cap\{k$, where $k$ is a natural number : $\left.\left(1+\left(i_{0}-^{\prime} 1\right) \cdot 8\right)+\left(j_{0}-^{\prime} 1\right) \cdot 32 \leqslant k \leqslant\left(8+\left(i_{0}-^{\prime} 1\right) \cdot 8\right)+\left(j_{0}-^{\prime} 1\right) \cdot 32\right\}=\emptyset$.

(17) Let us consider natural numbers $k, i, j, i_{0}, j_{0}$. Suppose

(i) $1 \leqslant k \leqslant 128$, and

(ii) $i, j, i_{0}, j_{0} \in \operatorname{Seg} 4$, and

(iii) $\left(1+\left(i-^{\prime} 1\right) \cdot 8\right)+\left(j-^{\prime} 1\right) \cdot 32 \leqslant k \leqslant\left(\left(1+\left(i-^{\prime} 1\right) \cdot 8\right)+\left(j-^{\prime} 1\right) \cdot 32\right)+7$, and 
(iv) $\left(1+\left(i_{0}-^{\prime} 1\right) \cdot 8\right)+\left(j_{0}-^{\prime} 1\right) \cdot 32 \leqslant k \leqslant\left(\left(1+\left(i_{0}-^{\prime} 1\right) \cdot 8\right)+\left(j_{0}-^{\prime} 1\right) \cdot 32\right)+7$.

Then

(v) $i=i_{0}$, and

(vi) $j=j_{0}$.

The theorem is a consequence of (16).

(18) The array of AES-State is one-to-one. The theorem is a consequence of (15). Proof: For every elements $x_{1}, x_{2}$ such that $x_{1}, x_{2} \in$ Boolean $^{128}$ and (the array of AES-State) $\left(x_{1}\right)=$ (the array of AES-State) $\left(x_{2}\right)$ holds $x_{1}=x_{2}$ by [15, (3)], [2, (11)], [4, (1)].

(19) The array of AES-State is onto. The theorem is a consequence of (15) and (17). Proof: For every element $y$ such that $y \in\left(\left(\text { Boolean }^{8}\right)^{4}\right)^{4}$ there exists an element $x$ such that $x \in$ Boolean $^{128}$ and $y=$ (the array of $\operatorname{AES}-\operatorname{State})(x)$ by [4, (1)], [7, (3)], [15, (3)].

Let us note that the array of AES-State is bijective.

Now we state the proposition:

(20) Let us consider an element $c$ of $\left(\left(\text { Boolean }^{8}\right)^{4}\right)^{4}$. Then (the array of AES-State $)\left((\text { the array of AES-State })^{-1}(c)\right)=c$.

\section{SubBytes}

In this paper $S$ denotes a permutation of Boolean ${ }^{8}$.

Let us consider $S$. The functor $\operatorname{SubBytes}(S)$ yielding a function from $\left(\left(\text { Boolean }^{8}\right)^{4}\right)^{4}$ into $\left(\left(\text { Boolean }^{8}\right)^{4}\right)^{4}$ is defined by

(Def. 2) Let us consider an element $i_{1}$ of $\left(\left(\text { Boolean }^{8}\right)^{4}\right)^{4}$ and natural numbers $i$, $j$. Suppose $i, j \in \operatorname{Seg} 4$. Then there exists an element $i_{2}$ of Boolean ${ }^{8}$ such that

(i) $i_{2}=i_{1}(i)(j)$, and

(ii) $i t\left(i_{1}\right)(i)(j)=S\left(i_{2}\right)$.

The functor InvSubBytes $(S)$ yielding a function from $\left(\left(\text { Boolean }^{8}\right)^{4}\right)^{4}$ into $\left(\left(\text { Boolean }^{8}\right)^{4}\right)^{4}$ is defined by

(Def. 3) Let us consider an element $i_{1}$ of $\left(\left(\text { Boolean }^{8}\right)^{4}\right)^{4}$ and natural numbers $i$, $j$. Suppose $i, j \in \operatorname{Seg} 4$. Then there exists an element $i_{2}$ of Boolean ${ }^{8}$ such that

(i) $i_{2}=i_{1}(i)(j)$, and

(ii) $i t\left(i_{1}\right)(i)(j)=S^{-1}\left(i_{2}\right)$.

Now we state the propositions: 
(21) Let us consider an element $i_{1}$ of $\left(\left(\text { Boolean }^{8}\right)^{4}\right)^{4}$.

Then $(\operatorname{InvSubBytes}(S))\left((\operatorname{SubBytes}(S))\left(i_{1}\right)\right)=i_{1}$. The theorem is a consequence of $(7)$.

(22) Let us consider an element $o$ of $\left(\left(\text { Boolean }^{8}\right)^{4}\right)^{4}$.

Then $(\operatorname{SubBytes}(S))((\operatorname{InvSubBytes}(S))(o))=o$. The theorem is a consequence of (7).

(23) (i) $\operatorname{SubBytes}(S)$ is one-to-one, and

(ii) $\operatorname{SubBytes}(S)$ is onto, and

(iii) $\operatorname{InvSubBytes}(S)$ is one-to-one, and

(iv) $\operatorname{InvSubBytes}(S)$ is onto, and

(v) $\operatorname{InvSubBytes}(S)=(\operatorname{SubBytes}(S))^{-1}$, and

(vi) $\operatorname{SubBytes}(S)=(\operatorname{InvSubBytes}(S))^{-1}$.

The theorem is a consequence of (21), (22), and (14).

\section{ShiftRows}

The functor ShiftRows yielding a function

from $\left(\left(\text { Boolean }^{8}\right)^{4}\right)^{4}$ into $\left(\left(\text { Boolean }^{8}\right)^{4}\right)^{4}$ is defined by

(Def. 4) Let us consider an element $i_{1}$ of $\left(\left(\text { Boolean }^{8}\right)^{4}\right)^{4}$ and a natural number $i$. Suppose $i \in \operatorname{Seg} 4$. Then there exists an element $x_{i}$ of $\left(\text { Boolean }^{8}\right)^{4}$ such that

(i) $x_{i}=i_{1}(i)$, and

(ii) $i t\left(i_{1}\right)(i)=$ Op-Shift $\left(x_{i}, 5-i\right)$.

The functor InvShiftRows yielding a function from $\left(\left(\text { Boolean }^{8}\right)^{4}\right)^{4}$ into $\left(\left(\text { Boolean }^{8}\right)^{4}\right)^{4}$ is defined by

(Def. 5) Let us consider an element $i_{1}$ of $\left(\left(\text { Boolean }^{8}\right)^{4}\right)^{4}$ and a natural number $i$. Suppose $i \in \operatorname{Seg} 4$. Then there exists an element $x_{i}$ of $\left(\text { Boolean }^{8}\right)^{4}$ such that

(i) $x_{i}=i_{1}(i)$, and

(ii) $i t\left(i_{1}\right)(i)=$ Op-Shift $\left(x_{i}, i-1\right)$.

Now we state the propositions:

(24) Let us consider an element $i_{1}$ of $\left(\left(\text { Boolean }^{8}\right)^{4}\right)^{4}$. Then InvShiftRows(ShiftRows $\left.\left(i_{1}\right)\right)=i_{1}$.

(25) Let us consider an element $o$ of $\left(\left(\text { Boolean }^{8}\right)^{4}\right)^{4}$. Then ShiftRows(InvShiftRows $(o))=o$.

(26) (i) ShiftRows is one-to-one, and

(ii) ShiftRows is onto, and 
(iii) InvShiftRows is one-to-one, and

(iv) InvShiftRows is onto, and

(v) InvShiftRows $=$ ShiftRows ${ }^{-1}$, and

(vi) ShiftRows $=$ InvShiftRows ${ }^{-1}$.

\section{AddRoundKey}

The functor AddRoundKey yielding a function from $\left(\left(\text { Boolean }^{8}\right)^{4}\right)^{4} \times\left(\left(\text { Boolean }^{8}\right)^{4}\right)^{4}$ into $\left(\left(\text { Boolean }^{8}\right)^{4}\right)^{4}$ is defined by

(Def. 6) Let us consider elements $t_{1}, k_{1}$ of $\left(\left(\text { Boolean }^{8}\right)^{4}\right)^{4}$ and natural numbers $i$, $j$. Suppose $i, j \in \operatorname{Seg} 4$. Then there exist elements $t_{2}, k_{2}$ of Boolean ${ }^{8}$ such that

(i) $t_{2}=t_{1}(i)(j)$, and

(ii) $k_{2}=k_{1}(i)(j)$, and

(iii) $i t\left(t_{1}, k_{1}\right)(i)(j)=\mathrm{Op}-\mathrm{XOR}\left(t_{2}, k_{2}\right)$.

\section{KEY EXPANSION}

Let us consider $S$. Let $x$ be an element of $\left(\text { Boolean }^{8}\right)^{4}$.

The functor $\operatorname{SubWord}(S, x)$ yielding an element of $\left(\text { Boolean }{ }^{8}\right)^{4}$ is defined by

(Def. 7) Let us consider an element $i$ of Seg 4. Then $i t(i)=S(x(i))$.

The functor RotWord $(x)$ yielding an element of $\left(\text { Boolean }^{8}\right)^{4}$ is defined by the term

(Def. 8) Op-LeftShift $x$.

Let $n, m$ be non zero elements of $\mathbb{N}$ and $s, t$ be elements of $\left(\text { Boolean }^{n}\right)^{m}$. The functor XOR-Word $(s, t)$ yielding an element of $\left(\text { Boolean }^{n}\right)^{m}$ is defined by

(Def. 9) Let us consider an element $i$ of Seg $m$. Then $i t(i)=\mathrm{Op}-\mathrm{XOR}(s(i), t(i))$.

The functor Rcon yielding an element of $\left(\left(\text { Boolean }^{8}\right)^{4}\right)^{10}$ is defined by

(Def. 10) (i) it $(1)=\langle\langle 0,0,0,0\rangle \frown\langle 0,0,0,1\rangle,\langle 0,0,0,0\rangle \frown\langle 0,0,0,0\rangle,\langle 0,0,0,0\rangle \frown\langle 0$, $0,0,0\rangle,\langle 0,0,0,0\rangle \frown\langle 0,0,0,0\rangle\rangle$, and

(ii) it $(2)=\langle\langle 0,0,0,0\rangle\urcorner\langle 0,0,1,0\rangle,\langle 0,0,0,0\rangle \frown\langle 0,0,0,0\rangle,\langle 0,0,0,0\rangle \frown\langle 0$, $0,0,0\rangle,\langle 0,0,0,0\rangle \frown\langle 0,0,0,0\rangle\rangle$, and

(iii) it $(3)=\langle\langle 0,0,0,0\rangle\urcorner\langle 0,1,0,0\rangle,\langle 0,0,0,0\rangle \frown\langle 0,0,0,0\rangle,\langle 0,0,0,0\rangle \frown\langle 0$, $0,0,0\rangle,\langle 0,0,0,0\rangle \frown\langle 0,0,0,0\rangle\rangle$, and

(iv) it $(4)=\left\langle\langle 0,0,0,0\rangle^{\frown}\langle 1,0,0,0\rangle,\langle 0,0,0,0\rangle^{\frown}\langle 0,0,0,0\rangle,\langle 0,0,0,0\rangle^{\frown}\langle 0\right.$, $0,0,0\rangle,\langle 0,0,0,0\rangle \frown\langle 0,0,0,0\rangle\rangle$, and 
(v) it $(5)=\left\langle\langle 0,0,0,1\rangle \frown\langle 0,0,0,0\rangle,\langle 0,0,0,0\rangle \frown\langle 0,0,0,0\rangle,\langle 0,0,0,0\rangle^{\frown}\langle 0\right.$, $0,0,0\rangle,\langle 0,0,0,0\rangle \frown\langle 0,0,0,0\rangle\rangle$, and

(vi) it $(6)=\langle\langle 0,0,1,0\rangle \frown\langle 0,0,0,0\rangle,\langle 0,0,0,0\rangle \frown\langle 0,0,0,0\rangle,\langle 0,0,0,0\rangle \frown\langle 0$, $0,0,0\rangle,\langle 0,0,0,0\rangle \frown\langle 0,0,0,0\rangle\rangle$, and

(vii) it $(7)=\langle\langle 0,1,0,0\rangle \frown\langle 0,0,0,0\rangle,\langle 0,0,0,0\rangle \frown\langle 0,0,0,0\rangle,\langle 0,0,0,0\rangle \frown\langle 0$, $0,0,0\rangle,\langle 0,0,0,0\rangle \frown\langle 0,0,0,0\rangle\rangle$, and

(viii) it $(8)=\langle\langle 1,0,0,0\rangle \frown\langle 0,0,0,0\rangle,\langle 0,0,0,0\rangle \frown\langle 0,0,0,0\rangle,\langle 0,0,0,0\rangle \frown\langle 0$, $0,0,0\rangle,\langle 0,0,0,0\rangle \frown\langle 0,0,0,0\rangle\rangle$, and

(ix) it $(9)=\langle\langle 0,0,0,1\rangle \frown\langle 1,0,1,1\rangle,\langle 0,0,0,0\rangle \frown\langle 0,0,0,0\rangle,\langle 0,0,0,0\rangle \frown\langle 0$, $0,0,0\rangle,\langle 0,0,0,0\rangle \frown\langle 0,0,0,0\rangle\rangle$, and

(x) it $(10)=\langle\langle 0,0,1,1\rangle \frown\langle 0,1,1,0\rangle,\langle 0,0,0,0\rangle \frown\langle 0,0,0,0\rangle,\langle 0,0,0,0\rangle\urcorner\langle 0$, $0,0,0\rangle,\langle 0,0,0,0\rangle \frown\langle 0,0,0,0\rangle\rangle$.

Let us consider $S$. Let $m, i$ be natural numbers and $w$ be an element of $\left(\text { Boolean }^{8}\right)^{4}$. Assume $m=4$ or $m=6$ or $m=8$ and $i<4 \cdot(7+m)$ and $m \leqslant i$. The functor KeyExpansionT $(S, m, i, w)$ yielding an element of $\left(\text { Boolean }^{8}\right)^{4}$ is defined by

(Def. 11) (i) there exists an element $T_{3}$ of $\left(\text { Boolean }^{8}\right)^{4} \operatorname{such}$ that $T_{3}=\operatorname{Rcon}\left(\frac{i}{m}\right)$ and $i t=\operatorname{XOR}-W o r d\left(\operatorname{SubWord}(S,(\operatorname{RotWord}(w))), T_{3}\right)$, if $i \bmod m=0$,

(ii) $i t=\operatorname{SubWord}(S, w)$, if $m=8$ and $i \bmod 8=4$,

(iii) $i t=w$, otherwise.

Let $m$ be a natural number. Assume $m=4$ or $m=6$ or $m=8$. The functor $\operatorname{KeyExpansionW}(S, m)$ yielding a function from $\left(\left(\text { Boolean }^{8}\right)^{4}\right)^{m}$ into $\left(\left(\text { Boolean }^{8}\right)^{4}\right)^{4 \cdot(7+m)}$ is defined by

(Def. 12) Let us consider an element $K$ of $\left(\left(\text { Boolean }^{8}\right)^{4}\right)^{m}$. Then

(i) for every element $i$ of $\mathbb{N}$ such that $i<m$ holds $i t(K)(i+1)=K(i+1)$, and

(ii) for every element $i$ of $\mathbb{N}$ such that $m \leqslant i<4 \cdot(7+m)$ there exists an element $P$ of $\left(\text { Boolean }^{8}\right)^{4}$ and there exists an element $Q$ of $\left(\text { Boolean }^{8}\right)^{4}$ such that $P=i t(K)((i-m)+1)$ and $Q=i t(K)(i)$ and $i t(K)(i+1)=\mathrm{XOR}-\operatorname{Word}(P,(\operatorname{KeyExpansionT}(S, m, i, Q)))$.

The functor KeyExpansion $(S, m)$ yielding a function from $\left(\left(\text { Boolean }^{8}\right)^{4}\right)^{m}$ into $\left(\left(\left(\text { Boolean }^{8}\right)^{4}\right)^{4}\right)^{7+m}$ is defined by

(Def. 13) Let us consider an element $K$ of $\left(\left(\text { Boolean }^{8}\right)^{4}\right)^{m}$. Then there exists an element $w$ of $\left(\left(\text { Boolean }^{8}\right)^{4}\right)^{4 \cdot(7+m)}$ such that

(i) $w=(\operatorname{KeyExpansionW}(S, m))(K)$, and

(ii) for every natural number $i$ such that $i<7+m$ holds $i t(K)(i+1)=$ $\langle w(4 \cdot i+1), w(4 \cdot i+2), w(4 \cdot i+3), w(4 \cdot i+4)\rangle$. 


\section{ENCRYPTION AND DECRYPTION}

In the sequel $\mathcal{M}_{1}$ denotes a permutation of $\left(\left(\text { Boolean }^{8}\right)^{4}\right)^{4}$ and $\mathcal{M}_{2}$ denotes a permutation of $\left(\left(\text { Boolean }^{8}\right)^{4}\right)^{4}$.

Let us consider $S$ and $\mathcal{M}_{1}$. Let $m$ be a natural number, $t_{1}$ be an element of $\left(\left(\text { Boolean }^{8}\right)^{4}\right)^{4}$, and $K$ be an element of $\left(\left(\text { Boolean }^{8}\right)^{4}\right)^{m}$. The functor $\operatorname{AES}-\operatorname{Cipher}\left(S, \mathcal{M}_{1}, t_{1}, K\right)$ yielding an element of $\left(\left(\text { Boolean }^{8}\right)^{4}\right)^{4}$ is defined by

(Def. 14) There exists a finite sequence $s_{1}$ of elements of $\left(\left(\text { Boolean }^{8}\right)^{4}\right)^{4}$ such that

(i) len $s_{1}=(7+m)-1$, and

(ii) there exists an element $K_{1}$ of $\left(\left(\text { Boolean }^{8}\right)^{4}\right)^{4}$ such that

$K_{1}=(\operatorname{KeyExpansion}(S, m))(K)(1)$ and $s_{1}(1)=\operatorname{AddRoundKey}\left(t_{1}, K_{1}\right)$, and

(iii) for every natural number $i$ such that $1 \leqslant i<(7+m)-1$ there exists an element $K_{i}$ of $\left(\left(\text { Boolean }^{8}\right)^{4}\right)^{4}$ such that

$K_{i}=(\operatorname{KeyExpansion}(S, m))(K)(i+1)$ and

$s_{1}(i+1)=$ AddRoundKey $\left(\left(\left(\mathcal{M}_{1} \cdot \operatorname{ShiftRows}\right) \cdot \operatorname{SubBytes}(S)\right)\left(s_{1}(i)\right), K_{i}\right)$, and

(iv) there exists an element $K_{n}$ of $\left(\left(\text { Boolean }^{8}\right)^{4}\right)^{4}$ such that

$K_{n}=(\operatorname{KeyExpansion}(S, m))(K)(7+m)$ and

$i t=$ AddRoundKey $\left((\operatorname{ShiftRows} \cdot \operatorname{SubBytes}(S))\left(s_{1}((7+m)-1)\right), K_{n}\right)$.

The functor AES-InvCipher $\left(S, \mathcal{M}_{1}, t_{1}, K\right)$ yielding an element of $\left(\left(\text { Boolean }^{8}\right)^{4}\right)^{4}$ is defined by

(Def. 15) There exists a finite sequence $s_{1}$ of elements of $\left(\left(\text { Boolean }^{8}\right)^{4}\right)^{4}$ such that

(i) len $s_{1}=(7+m)-1$, and

(ii) there exists an element $K_{1}$ of $\left(\left(\text { Boolean }^{8}\right)^{4}\right)^{4}$ such that

$K_{1}=(\operatorname{Rev}((\operatorname{KeyExpansion}(S, m))(K)))(1)$ and $s_{1}(1)=$

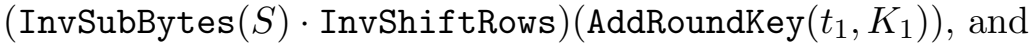

(iii) for every natural number $i$ such that $1 \leqslant i<(7+m)-1$ there exists an element $K_{i}$ of $\left(\left(\text { Boolean }^{8}\right)^{4}\right)^{4}$ such that

$K_{i}=(\operatorname{Rev}((\operatorname{KeyExpansion}(S, m))(K)))(i+1)$ and $s_{1}(i+1)=$ $\left((\operatorname{InvSubBytes}(S) \cdot \operatorname{InvShiftRows}) \cdot \mathcal{M}_{1}^{-1}\right)\left(\operatorname{AddRoundKey}\left(s_{1}(i), K_{i}\right)\right)$, and

(iv) there exists an element $K_{n}$ of $\left(\left(\text { Boolean }^{8}\right)^{4}\right)^{4}$ such that $K_{n}=(\operatorname{Rev}((\operatorname{KeyExpansion}(S, m))(K)))(7+m)$ and $i t=$ $\operatorname{AddRoundKey}\left(s_{1}((7+m)-1), K_{n}\right)$.

Now we state the propositions:

(27) Let us consider an element $i_{1}$ of $\left(\left(\text { Boolean }^{8}\right)^{4}\right)^{4}$.

Then $\mathcal{M}_{1}^{-1}\left(\mathcal{M}_{1}\left(i_{1}\right)\right)=i_{1}$.

(28) Let us consider an element $o$ of $\left(\left(\text { Boolean }^{8}\right)^{4}\right)^{4}$. Then $\mathcal{M}_{1}\left(\mathcal{M}_{1}{ }^{-1}(o)\right)=o$. 
Let us consider a natural number $m$ and an element $t_{1}$ of $\left(\left(\text { Boolean }^{8}\right)^{4}\right)^{4}$. Now we state the propositions:

(29) $(\operatorname{InvSubBytes}(S) \cdot \operatorname{InvShiftRows})\left((\operatorname{ShiftRows} \cdot \operatorname{SubBytes}(S))\left(t_{1}\right)\right)=t_{1}$.

(30) $\left((\right.$ InvSubBytes $(S) \cdot$ InvShiftRows $\left.) \cdot \mathcal{M}_{1}{ }^{-1}\right)\left(\left(\left(\mathcal{M}_{1} \cdot\right.\right.\right.$ ShiftRows $) \cdot$ SubBytes $\left.(S))\left(t_{1}\right)\right)=t_{1}$.

Now we state the propositions:

(31) Let us consider a natural number $m$, an element $t_{1}$ of $\left(\left(\text { Boolean }^{8}\right)^{4}\right)^{4}$, an element $K$ of $\left(\left(\text { Boolean }^{8}\right)^{4}\right)^{m}$, and elements $d_{k}, e_{k}$ of $\left(\left(\text { Boolean }^{8}\right)^{4}\right)^{4}$. Suppose

(i) $m=4$ or $m=6$ or $m=8$, and

(ii) $d_{k}=(\operatorname{Rev}((\operatorname{KeyExpansion}(S, m))(K)))(1)$, and

(iii) $e_{k}=(\operatorname{KeyExpansion}(S, m))(K)(7+m)$.

Then AddRoundKey $\left(\operatorname{AddRoundKey}\left(t_{1}, e_{k}\right), d_{k}\right)=t_{1}$. The theorem is a consequence of $(7)$.

(32) Let us consider a natural number $m$, an element $t_{1}$ of $\left(\left(\text { Boolean }^{8}\right)^{4}\right)^{4}$, an element $k_{1}$ of $\left(\left(\text { Boolean }^{8}\right)^{4}\right)^{m}$, and elements $d_{k}, e_{k}$ of $\left(\left(\text { Boolean }^{8}\right)^{4}\right)^{4}$. Suppose

(i) $m=4$ or $m=6$ or $m=8$, and

(ii) $d_{k}=(\operatorname{KeyExpansion}(S, m))\left(k_{1}\right)(1)$, and

(iii) $e_{k}=\left(\operatorname{Rev}\left((\operatorname{KeyExpansion}(S, m))\left(k_{1}\right)\right)\right)(7+m)$.

Then AddRoundKey(AddRoundKey $\left.\left(t_{1}, e_{k}\right), d_{k}\right)=t_{1}$. The theorem is a consequence of $(7)$.

(33) Let us consider a natural number $m$, elements $t_{1}, o_{1}$ of $\left(\left(\text { Boolean }^{8}\right)^{4}\right)^{4}$, an element $K$ of $\left(\left(\text { Boolean }^{8}\right)^{4}\right)^{m}$, and elements $K_{1}, K_{n}$ of $\left(\left(\text { Boolean }^{8}\right)^{4}\right)^{4}$. Suppose

(i) $m=4$ or $m=6$ or $m=8$, and

(ii) $K_{1}=(\operatorname{KeyExpansion}(S, m))(K)(1)$, and

(iii) $K_{n}=(\operatorname{Rev}((\operatorname{KeyExpansion}(S, m))(K)))(7+m)$, and

(iv) $o_{1}=\operatorname{AddRoundKey}\left((\operatorname{ShiftRows} \cdot \operatorname{SubBytes}(S))\left(t_{1}\right), K_{n}\right)$.

Then $(\operatorname{InvSubBytes}(S) \cdot \operatorname{InvShiftRows})\left(\operatorname{AddRoundKey}\left(o_{1}, K_{1}\right)\right)=t_{1}$. The theorem is a consequence of (32) and (29).

(34) Let us consider natural numbers $m, i$, an element $t_{1}$ of $\left(\left(\text { Boolean }^{8}\right)^{4}\right)^{4}$, an element $K$ of $\left(\left(\text { Boolean }^{8}\right)^{4}\right)^{m}$, and elements $e_{i}, d_{i}$ of $\left(\left(\text { Boolean }^{8}\right)^{4}\right)^{4}$. Suppose

(i) $m=4$ or $m=6$ or $m=8$, and

(ii) $i \leqslant(7+m)-1$, and

(iii) $e_{i}=(\operatorname{KeyExpansion}(S, m))(K)((7+m)-i)$, and 
(iv) $d_{i}=(\operatorname{Rev}((\operatorname{KeyExpansion}(S, m))(K)))(i+1)$.

Then AddRoundKey(AddRoundKey $\left.\left(t_{1}, e_{i}\right), d_{i}\right)=t_{1}$. The theorem is a consequence of $(7)$.

(35) Let us consider a natural number $m$, an element $t_{1}$ of $\left(\left(\text { Boolean }^{8}\right)^{4}\right)^{4}$, and an element $K$ of $\left(\left(\text { Boolean }^{8}\right)^{4}\right)^{m}$. Suppose

(i) $m=4$, or

(ii) $m=6$, or

(iii) $m=8$.

Then AES-InvCipher $\left(S, \mathcal{M}_{1},\left(\operatorname{AES}-\operatorname{Cipher}\left(S, \mathcal{M}_{1}, t_{1}, K\right)\right), K\right)=t_{1}$. The theorem is a consequence of (34) and (30). Proof: Reconsider $N=$ $(7+m)-1$ as a natural number. Consider $e_{s}$ being a finite sequence of elements of $\left(\left(\text { Boolean }^{8}\right)^{4}\right)^{4}$ such that len $e_{s}=N$ and there exists an element $K_{1}$ of $\left(\left(\text { Boolean }^{8}\right)^{4}\right)^{4}$ such that $K_{1}=(\operatorname{KeyExpansion}(S, m))(K)(1)$ and $e_{s}(1)=\operatorname{AddRoundKey}\left(t_{1}, K_{1}\right)$ and for every natural number $i$ such that $1 \leqslant i<N$ there exists an element $K_{i}$ of $\left(\left(\text { Boolean }^{8}\right)^{4}\right)^{4}$ such that $K_{i}=(\operatorname{KeyExpansion}(S, m))(K)(i+1)$ and $e_{s}(i+1)=\operatorname{AddRoundKey}\left(\left(\left(\mathcal{M}_{1}\right.\right.\right.$. ShiftRows $\left.) \cdot \operatorname{SubBytes}(S))\left(e_{s}(i)\right), K_{i}\right)$ and there exists an element $K_{n}$ of $\left(\left(\text { Boolean }^{8}\right)^{4}\right)^{4}$ such that $K_{n}=(\operatorname{KeyExpansion}(S, m))(K)(7+m)$ and $\operatorname{AES-Cipher}\left(S, \mathcal{M}_{1}, t_{1}, K\right)=\operatorname{AddRoundKey}\left((\operatorname{ShiftRows} \cdot \operatorname{SubBytes}(S))\left(e_{s}\right.\right.$ $\left.(N)), K_{n}\right)$. Consider $d_{s}$ being a finite sequence of elements of $\left(\left(\text { Boolean }^{8}\right)^{4}\right)^{4}$ such that len $d_{s}=N$ and there exists an element $K_{1}$ of $\left(\left(\text { Boolean }^{8}\right)^{4}\right)^{4}$ such that $K_{1}=(\operatorname{Rev}((\operatorname{KeyExpansion}(S, m))(K)))(1)$ and $d_{s}(1)=($ InvSubBytes $(S)$-InvShiftRows)(AddRoundKey(AES-Cipher $\left.\left(S, \mathcal{M}_{1}, t_{1}, K\right), K_{1}\right)$ ) and for every natural number $i$ such that $1 \leqslant i<N$ there exists an element $K_{i}$ of $\left(\left(\text { Boolean }^{8}\right)^{4}\right)^{4}$ such that $K_{i}=(\operatorname{Rev}((\operatorname{KeyExpansion}(S, m))(K)))(i+1)$ and $d_{s}(i+1)=\left((\operatorname{InvSubBytes}(S) \cdot \operatorname{InvShiftRows}) \cdot \mathcal{M}_{1}{ }^{-1}\right)\left(\operatorname{AddRoundKey}\left(d_{s}\right.\right.$ $\left.\left.(i), K_{i}\right)\right)$ and there exists an element $K_{n}$ of $\left(\left(\text { Boolean }^{8}\right)^{4}\right)^{4}$ such that $K_{n}=$ $(\operatorname{Rev}((\operatorname{KeyExpansion}(S, m))(K)))(7+m)$ and $\operatorname{AES}-\operatorname{Inv} \operatorname{Cipher}\left(S, \mathcal{M}_{1}\right.$, $\left.\left(\operatorname{AES}-\operatorname{Cipher}\left(S, \mathcal{M}_{1}, t_{1}, K\right)\right), K\right)=\operatorname{AddRoundKey}\left(d_{s}(N), K_{n}\right)$. Consider $e_{1}$ being an element of $\left(\left(\text { Boolean }^{8}\right)^{4}\right)^{4}$ such that $e_{1}=(\operatorname{KeyExpansion}(S, m))$ $(K)(1)$ and $e_{s}(1)=\operatorname{AddRoundKey}\left(t_{1}, e_{1}\right)$. Consider $e_{n}$ being an element of $\left(\left(\text { Boolean }^{8}\right)^{4}\right)^{4}$ such that $e_{n}=(\operatorname{KeyExpansion}(S, m))(K)(7+m)$ and $\operatorname{AES-Cipher}\left(S, \mathcal{M}_{1}, t_{1}, K\right)=\operatorname{AddRoundKey}\left((\operatorname{ShiftRows} \cdot \operatorname{SubBytes}(S))\left(e_{s}\right.\right.$ $\left.(N)), e_{n}\right)$. Consider $d_{1}$ being an element of $\left(\left(\text { Boolean }^{8}\right)^{4}\right)^{4}$ such that $d_{1}=$ $(\operatorname{Rev}((\operatorname{KeyExpansion}(S, m))(K)))(1)$ and $d_{s}(1)=(\operatorname{InvSubBytes}(S)$. InvShiftRows)(AddRoundKey(AES-Cipher $\left.\left.\left(S, \mathcal{M}_{1}, t_{1}, K\right), d_{1}\right)\right)$. Consider $d_{n}$ being an element of $\left(\left(\text { Boolean }^{8}\right)^{4}\right)^{4}$ such that $d_{n}=(\operatorname{Rev}((\operatorname{KeyExpansion}(S$, $m)(K)))(7+m)$ and AES-InvCipher $\left(S, \mathcal{M}_{1},\left(\operatorname{AES}-\operatorname{Cipher}\left(S, \mathcal{M}_{1}, t_{1}, K\right)\right)\right.$, $K)=\operatorname{AddRoundKey}\left(d_{s}(N), d_{n}\right)$. Define $\mathcal{R}$ [natural number] $\equiv$ if $\$_{1}<N$, then $d_{s}\left(\$_{1}+1\right)=e_{s}\left(N-\$_{1}\right)$. For every natural number $i$ such that $\mathcal{R}[i]$ 
holds $\mathcal{R}[i+1]$ by [2, (11)], [15, (3)], [2, (14)]. For every natural number $k$, $\mathcal{R}[k]$ from [2, Sch. 2].

(36) Let us consider a non empty set $D$, non zero elements $n, m$ of $\mathbb{N}$, and an element $r$ of $D^{n}$. Suppose

(i) $m \leqslant n$, and

(ii) $8 \leqslant n-m$.

Then Op-Left(Op-Right $(r, m), 8)$ is an element of $D^{8}$.

Let $r$ be an element of Boolean ${ }^{128}$. The functor AES-InitState128Key $(r)$ yielding an element of $\left(\left(\text { Boolean }^{8}\right)^{4}\right)^{4}$ is defined by

(Def. 16) (i) it $(1)=\langle$ Op-Left $(r, 8)$, Op-Left $($ Op-Right $(r, 8), 8)$, Op-Left $($ Op-Right $(r, 16), 8)$, Op-Left $(O p-\operatorname{Right}(r, 24), 8)\rangle$, and

(ii) it $(2)=\langle$ Op-Left $($ Op-Right $(r, 32), 8)$, Op-Left $(O p-\operatorname{Right}(r, 40), 8)$, Op-Left $(\mathrm{Op}-\operatorname{Right}(r, 48), 8)$, Op-Left $(\mathrm{Op}-\operatorname{Right}(r, 56), 8)\rangle$, and

(iii) it $(3)=\langle$ Op-Left $($ Op-Right $(r, 64), 8)$, Op-Left $($ Op-Right $(r, 72), 8)$, Op-Left $(\mathrm{Op}-\operatorname{Right}(r, 80), 8)$, Op-Left $(\mathrm{Op}-\operatorname{Right}(r, 88), 8)\rangle$, and

(iv) $i t(4)=\langle$ Op-Left $($ Op-Right $(r, 96), 8)$, Op-Left $($ Op-Right $(r, 104), 8)$, Op-Left $(O p-\operatorname{Right}(r, 112), 8)$, Op-Right $(r, 120)\rangle$.

Let $r$ be an element of Boolean ${ }^{192}$. The functor AES-InitState192Key $(r)$ yielding an element of $\left(\left(\text { Boolean }^{8}\right)^{4}\right)^{6}$ is defined by

(Def. 17) (i) $i t(1)=\langle$ Op-Left $(r, 8)$, Op-Left $($ Op-Right $(r, 8), 8)$, Op-Left $($ Op-Right $(r, 16), 8)$, Op-Left $(\mathrm{Op}-\operatorname{Right}(r, 24), 8)\rangle$, and

(ii) $i t(2)=\langle$ Op-Left $($ Op-Right $(r, 32), 8)$, Op-Left $($ Op-Right $(r, 40), 8)$, Op-Left(Op-Right $(r, 48), 8)$, Op-Left $(\mathrm{Op}-\operatorname{Right}(r, 56), 8)\rangle$, and

(iii) $i t(3)=\langle$ Op-Left $($ Op-Right $(r, 64), 8)$, Op-Left $($ Op-Right $(r, 72), 8)$, Op-Left $(\mathrm{Op}-\operatorname{Right}(r, 80), 8)$, Op-Left $(\mathrm{Op}-\operatorname{Right}(r, 88), 8)\rangle$, and

(iv) $i t(4)=\langle$ Op-Left $($ Op-Right $(r, 96), 8)$, Op-Left $($ Op-Right $(r, 104), 8)$, Op-Left(Op-Right $(r, 112), 8)$, Op-Left $(O p-\operatorname{Right}(r, 120), 8)\rangle$, and

(v) it $(5)=\langle$ Op-Left $(O p-\operatorname{Right}(r, 128), 8)$, Op-Left $($ Op-Right $(r, 136), 8)$, Op-Left $(O p-\operatorname{Right}(r, 144), 8)$, Op-Left $(O p-\operatorname{Right}(r, 152), 8)\rangle$, and

(vi) $i t(6)=\langle$ Op-Left $($ Op-Right $(r, 160), 8)$, Op-Left $($ Op-Right $(r, 168), 8)$, Op-Left $($ Op-Right $(r, 176), 8)$, Op-Right $(r, 184)\rangle$.

Let $r$ be an element of Boolean ${ }^{256}$. The functor AES-InitState256Key $(r)$ yielding an element of $\left(\left(\text { Boolean }^{8}\right)^{4}\right)^{8}$ is defined by

(Def. 18) (i) $i t(1)=\langle$ Op-Left $(r, 8)$, Op-Left $($ Op-Right $(r, 8), 8)$, Op-Left (Op-Right $(r, 16), 8)$, Op-Left $(O p-\operatorname{Right}(r, 24), 8)\rangle$, and

(ii) $i t(2)=\langle$ Op-Left $($ Op-Right $(r, 32), 8)$, Op-Left $($ Op-Right $(r, 40), 8)$, Op-Left $($ Op-Right $(r, 48), 8)$, Op-Left $($ Op-Right $(r, 56), 8)\rangle$, and 
(iii) $i t(3)=\langle$ Op-Left $($ Op-Right $(r, 64), 8)$, Op-Left $($ Op-Right $(r, 72), 8)$, Op-Left $(\mathrm{Op}-\operatorname{Right}(r, 80), 8)$, Op-Left $(\mathrm{Op}-\operatorname{Right}(r, 88), 8)\rangle$, and

(iv) $i t(4)=\langle$ Op-Left $($ Op-Right $(r, 96), 8)$, Op-Left $($ Op-Right $(r, 104), 8)$, Op-Left(Op-Right $(r, 112), 8)$, Op-Left(Op-Right $(r, 120), 8)\rangle$, and

(v) $i t(5)=\langle$ Op-Left $(O p-\operatorname{Right}(r, 128), 8)$, Op-Left $($ Op-Right $(r, 136), 8)$, Op-Left(Op-Right $(r, 144), 8)$, Op-Left (Op-Right $(r, 152), 8)\rangle$, and

(vi) it $(6)=\langle$ Op-Left $($ Op-Right $(r, 160), 8)$, Op-Left $($ Op-Right $(r, 168), 8)$, Op-Left(Op-Right $(r, 176), 8)$, Op-Left $(O p-\operatorname{Right}(r, 184), 8)\rangle$, and

(vii) $i t(7)=\langle$ Op-Left $($ Op-Right $(r, 192), 8)$, Op-Left $($ Op-Right $(r, 200), 8)$, Op-Left $(O p-\operatorname{Right}(r, 208), 8)$, Op-Left $(O p-\operatorname{Right}(r, 216), 8)\rangle$, and

(viii) it $(8)=\langle$ Op-Left $($ Op-Right $(r, 224), 8)$, Op-Left $($ Op-Right $(r, 232), 8)$, Op-Left $($ Op-Right $(r, 240), 8)$, Op-Right $(r, 248)\rangle$.

Let us consider $S$ and $\mathcal{M}_{2}$. Let $m_{1}$ be an element of Boolean ${ }^{128}$ and $K$ be an element of Boolean ${ }^{128}$. The functor AES-128enc $\left(S, \mathcal{M}_{2}, m_{1}, K\right)$ yielding an element of Boolean ${ }^{128}$ is defined by the term

(Def. 19) (The array of AES-State) $)^{-1}$ (AES-Cipher $\left(S, \mathcal{M}_{2},((\right.$ the array of AES-State $\left.\left.\left.\left(m_{1}\right)\right),(\operatorname{AES}-I n i t S t a t e 128 \operatorname{Key}(K))\right)\right)$.

Let $c$ be an element of Boolean ${ }^{128}$. The functor $\operatorname{AES}-128 \operatorname{dec}\left(S, \mathcal{M}_{2}, c, K\right)$ yielding an element of Boolean ${ }^{128}$ is defined by the term

(Def. 20) (The array of AES-State) $)^{-1}$ (AES-InvCipher $\left(S, \mathcal{M}_{2}\right.$, ((the array of AES-State $(c)),(\operatorname{AES}-\operatorname{InitState} 128 \operatorname{Key}(K))))$.

Now we state the proposition:

(37) Let us consider a permutation $S$ of Boolean $^{8}$, a permutation $\mathcal{M}_{2}$ of $\left(\left(\text { Boolean }^{8}\right)^{4}\right)^{4}$, and elements $m_{1}, K$ of Boolean ${ }^{128}$.

Then $\operatorname{AES}-128 \operatorname{dec}\left(S, \mathcal{M}_{2},\left(\operatorname{AES}-128 \operatorname{enc}\left(S, \mathcal{M}_{2}, m_{1}, K\right)\right), K\right)=m_{1}$. The theorem is a consequence of (20) and (35).

Let us consider $S$ and $\mathcal{M}_{2}$. Let $m_{1}$ be an element of Boolean ${ }^{128}$ and $K$ be an element of Boolean ${ }^{192}$. The functor AES-192enc $\left(S, \mathcal{M}_{2}, m_{1}, K\right)$ yielding an element of Boolean ${ }^{128}$ is defined by the term

(Def. 21) (The array of AES-State) $)^{-1}\left(\operatorname{AES}-\operatorname{Cipher}\left(S, \mathcal{M}_{2},((\right.\right.$ the array of AES-State $\left.\left.\left.\left(m_{1}\right)\right),(\operatorname{AES}-I n i t S t a t e 192 \operatorname{Key}(K))\right)\right)$.

Let $c$ be an element of Boolean ${ }^{128}$. The functor $\operatorname{AES}-192 \operatorname{dec}\left(S, \mathcal{M}_{2}, c, K\right)$ yielding an element of Boolean ${ }^{128}$ is defined by the term

(Def. 22) (The array of AES-State) ${ }^{-1}$ (AES-InvCipher $\left(S, \mathcal{M}_{2}\right.$, ((the array of AES-State $(c)),($ AES-InitState192Key $(K))))$.

Now we state the proposition:

(38) Let us consider a permutation $S$ of Boolean $^{8}$, a permutation $\mathcal{M}_{2}$ of $\left(\left(\text { Boolean }^{8}\right)^{4}\right)^{4}$, an element $m_{1}$ of Boolean ${ }^{128}$, and an element $K$ of Boolean ${ }^{192}$. 
Then AES-192dec $\left(S, \mathcal{M}_{2},\left(\operatorname{AES}-192 \operatorname{enc}\left(S, \mathcal{M}_{2}, m_{1}, K\right)\right), K\right)=m_{1}$. The theorem is a consequence of $(20)$ and (35).

Let us consider $S$ and $\mathcal{M}_{2}$. Let $m_{1}$ be an element of Boolean ${ }^{128}$ and $K$ be an element of Boolean ${ }^{256}$. The functor $\operatorname{AES}-256 \operatorname{enc}\left(S, \mathcal{M}_{2}, m_{1}, K\right)$ yielding an element of Boolean ${ }^{128}$ is defined by the term

(Def. 23) (The array of AES-State $)^{-1}$ (AES-Cipher $\left(S, \mathcal{M}_{2},((\right.$ the array of AES-State $\left.\left(m_{1}\right)\right),($ AES-InitState256Key $\left.\left.(K))\right)\right)$.

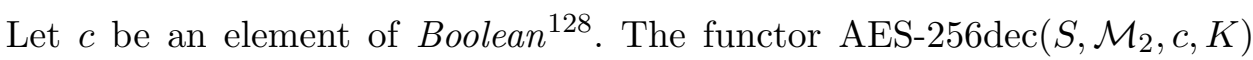
yielding an element of Boolean ${ }^{128}$ is defined by the term

(Def. 24) (The array of AES-State) ${ }^{-1}$ (AES-InvCipher $\left(S, \mathcal{M}_{2}\right.$, ((the array of AES-State $(c)),($ AES-InitState256Key $(K))))$.

Now we state the proposition:

(39) Let us consider a permutation $S$ of Boolean ${ }^{8}$, a permutation $\mathcal{M}_{2}$ of $\left(\left(\text { Boolean }^{8}\right)^{4}\right)^{4}$, an element $m_{1}$ of Boolean ${ }^{128}$, and an element $K$ of Boolean ${ }^{256}$.

Then $\operatorname{AES}-256 \operatorname{dec}\left(S, \mathcal{M}_{2},\left(\operatorname{AES}-256 \operatorname{enc}\left(S, \mathcal{M}_{2}, m_{1}, K\right)\right), K\right)=m_{1}$. The theorem is a consequence of $(20)$ and (35).

\section{REFERENCES}

[1] Grzegorz Bancerek. Cardinal numbers. Formalized Mathematics, 1(2):377-382, 1990.

[2] Grzegorz Bancerek. The fundamental properties of natural numbers. Formalized Mathematics, 1(1):41-46, 1990.

[3] Grzegorz Bancerek. The ordinal numbers Formalized Mathematics, 1(1):91-96, 1990.

[4] Grzegorz Bancerek and Krzysztof Hryniewiecki. Segments of natural numbers and finite sequences Formalized Mathematics, 1(1):107-114, 1990.

[5] Czesław Byliński. Binary operations. Formalized Mathematics, 1(1):175-180, 1990.

[6] Czesław Byliński. Finite sequences and tuples of elements of a non-empty sets Formalized Mathematics, 1(3):529-536, 1990.

[7] Czesław Byliński. Functions and their basic properties Formalized Mathematics, 1(1): 55-65, 1990.

[8] Czesław Byliński. Functions from a set to a set, Formalized Mathematics, 1(1):153-164, 1990.

[9] Czesław Byliński. Partial functions. Formalized Mathematics, 1(2):357-367, 1990.

[10] Czesław Byliński. Some basic properties of sets. Formalized Mathematics, 1(1):47-53, 1990.

[11] Agata Darmochwał. Finite sets Formalized Mathematics, 1(1):165-167, 1990.

[12] U.S. Department of Commerce/National Institute of Standards and Technology. FIPS PUB 197, Advanced Encryption Standard (AES), Federal Information Processing Standars Publicatıon, 2001.

[13] Hiroyuki Okazaki and Yasunari Shidama. Formalization of the data encryption standard. Formalized Mathematics, 20(2):125-146, 2012. doi:10.2478/v10037-012-0016-y.

[14] Andrzej Trybulec. On the decomposition of finite sequences Formalized Mathematics, 5 (3):317-322, 1996.

[15] Michał J. Trybulec. Integers Formalized Mathematics, 1(3):501-505, 1990.

[16] Wojciech A. Trybulec. Pigeon hole principle Formalized Mathematics, 1(3):575-579, 1990.

[17] Zinaida Trybulec. Properties of subsets. Formalized Mathematics, 1(1):67-71, 1990. 
[18] Edmund Woronowicz. Many argument relations. Formalized Mathematics, 1(4):733-737, 1990.

[19] Edmund Woronowicz. Relations and their basic properties. Formalized Mathematics, 1 (1):73-83, 1990.

Received October 7, 2013 